\title{
ICoNOs MM: The IT-Enabled Collaborative Networked Organizations Maturity Model
}

\author{
Roberto Santana Tapia* \\ Department of Computer Science \\ University of Twente \\ P.O. Box 217, 7500 AE Enschede, The Netherlands \\ r.santanatapia@utwente.nl
}

\begin{abstract}
The focus of this paper is to introduce a comprehensive model for assessing and improving maturity of business-IT alignment (B-ITa) in collaborative networked organizations (CNOs): the ICoNOs MM. This two dimensional maturity model (MM) addresses five levels of maturity as well as four domains to which these levels apply: partnering structure, information system (IS) architecture, process architecture and coordination. The model can be used to benchmark and support continuous improvement of B-ITa process areas in CNOs.
\end{abstract}

\section{Introduction}

B-ITa has been a concern in practice for several decades and has been studied by researchers for more than 15 years [1]. However, despite years of research, B-ITa still ranks as a major modern-day area of concern for both business practitioners and researchers. Interest in B-ITa is stimulated by cases of organizations that have successfully aligned their IT to gain competitive advantage [2, 3] and to improve organizational performance [4].

There is a considerable literature on B-ITa in single organizations. Within this broad scope of literature, a number of authors have stressed the importance to assess B-ITa in order to plan B-ITa improvement actions $[5,6,7,8]$. In support of this, these authors have developed MMs. MMs describe the development of a specific domain over time. Based on maturity assessments, organizations know whether a specific area is sufficiently refined and documented so that the activities in such area now have the potential to achieve its desired outcomes.

However, B-ITa in CNOs has hardly been studied. Yet, the problem is important because improved B-ITa entails a more efficient use of IT in the CNOs supporting the integration of ISs and processes across organizational boundaries. CNOs form the core of a new discipline [9] that focuses on the structure, behavior, and dynamics of networks of independent organizations that collaborate to better achieve common goals. We define a CNO to be any "mix-and-match" network of profit-and-loss responsible organizational units, or of independent organizations, connected by IT, that

* Supported by the Netherlands Organization for Scientific Research under contract number 638.003.407 (Value-Based Business-IT Alignment). 
work together to jointly accomplish tasks, reach common goals and serve customers over a period of time [10].

In several case studies $[11,12,13,14]$, we have witnessed that achieving B-ITa in CNOs is more complex than in single organizations because in such settings, B-ITa is driven by goals of different independent organizations commonly with no centralized decision-making processes. In our research, we have developed a MM that specifically addresses the processes needed for achieving alignment between business and IT in CNOs: the ICoNOs MM. This MM presents a roll up of recommendations - e.g., coordination mechanisms, implementation strategies and organizational changes, in the form of process areas, specific goals and specific practices. The ICoNOs MM has been designed and validated based on results of conceptual and empirical research activities. These research activities, and their results, have been progressively made public in several publications as presented in Table 1.

Table 1. Research activities and publications

\begin{tabular}{|l|l|l|}
\cline { 2 - 3 } \multicolumn{1}{c|}{} & Activity & Publication \\
\hline Goals and research approach & & {$[15]$} \\
\hline Initial attempts to identify the domains & Focus group & {$[16]$} \\
\cline { 2 - 3 } & Case study & \\
\hline MM design challenges and solutions & Literature survey & {$[17]$} \\
\hline Domains validation and B-ITa principles & Case studies & {$[11,12]$} \\
\hline First version of the process areas & Literature survey & {$[18]$} \\
\hline $\begin{array}{l}\text { Process areas validation and B-ITa best } \\
\text { practices }\end{array}$ & Case study & {$[13,14]$} \\
\hline $\begin{array}{l}\text { Specific goals and practices of the partner- } \\
\text { ing structure and the coordination domains }\end{array}$ & & \\
\hline
\end{tabular}

\section{Model Dimensions}

The ICoNOs MM is two-dimensional: the first dimension represents the number of discrete levels of maturity, whereas the second dimension represents the domains to which the levels apply. Domains are sets of process areas that share common knowledge of aspects within the scope of the MM (in our case, a domain is a group of processes which need to be performed by CNOs in their efforts for improving B-ITa - we call them 'B-ITa domains').

\subsection{The B-ITa Levels}

To maintain adherence to the CMMI (http://www.sei.cmu.edu/cmmi/), which is an internationally well-known MM, we decided to base the levels of the ICoNOs MM on it. Since our MM is a continuous MM in architecture [17], it is expected that our model incorporates six levels of maturity - as the CMMI does in its continuous representation. However, because we want to offer a roadmap to approach B-ITa process improvement (i.e., series of maturity levels) focusing on a set of B-ITa process areas that provide CNOs with specific practices characterized by each maturity level, we decided to include only five levels of maturity in the ICoNOs MM (as in the staged representation of the CMMI). That is, when using the ICoNOs MM, process improvement results 
are summarized in a single maturity level number in each of the B-ITa domains that the model includes. In our model, levels are used to describe an improvement path recommended for a CNO that wants to improve processes to achieve B-ITa. To reach a particular level, a CNO must satisfy all the set of process areas that are targeted for improvement in a particular B-ITa domain. The levels are:

- Level 1: Incomplete. At maturity level 1, processes related to a particular B-ITa domain are usually not performed or partially performed. It means such a particular domain is not explicitly considered when a CNO strives for B-ITa. Therefore, this level contains no processes in the ICoNOs MM.

- Level 2: Isolated. At maturity level 2, processes are the basic infrastructure in place to support a particular B-ITa domain. They (i) are planned and executed in accordance with a policy; (ii) employ skilled people who have adequate resources to produce controlled outputs; (iii) are monitored, controlled, and reviewed. However, such processes are isolated initiatives that are not managed from the entire CNO perspective.

- Level 3: Standardized. At maturity level 3, processes are directed to make improvements in the standardization and management of a particular B-ITa domain. Processes are performed from a CNO perspective (i.e., they are cooperative initiatives). They are well characterized and understood, and are described in standards, procedures, tools, and methods.

- Level 4: Quantitatively Managed. At maturity level 4, processes use statistical and other quantitative techniques. Quantitative objectives for quality and process performance are established and used as criteria in managing the process. Quality and process performance is understood in statistical terms and is managed throughout the life of the process.

- Level 5: Optimized. At maturity level 5, processes are improved based on an understanding of the common causes of variations inherent in the process. The focus of an optimized process is on continuously optimizing the range of process performance through both incremental and innovative improvements.

\subsection{The B-ITa Domains}

After conducting case studies in real-life CNOs to identify and validate the B-ITa domains, the ICoNOs MM includes the following four B-ITa domains:

Partnering structure, defined as the cross-organizational work division, organizational structure, and roles and responsibilities definition that indicate where and how the work gets done and who is involved. It helps to organize the collaborative work in the $\mathrm{CNO}$.

IS architecture, defined as the fundamental organization of the information management function of the participating organizations embodied in the information systems, i.e., software applications, that realize this function, their relationships to each other and to the environment, and the principles guiding its design and evolution - based on IEEE 1471 [20]. It must be noted that, in our work, we distinguish IS architecture from IT architecture. For us, IT architecture consists of the (i) the implementation platform, i.e., the collection of standard general-purpose software 
needed to run the IS architecture. It ranges from operating systems, middleware, network software to database management software; and the (ii) physical network, i.e., the physical resources that run software applications. This includes computers, cables, and user interface devices to support the running of the IS architecture.

Process architecture, defined as the choreography of all processes needed to reach the shared goals of the participating organizations. These processes are both primary business processes of the $\mathrm{CNO}$ and processes needed for information exchange.

Coordination, defined as the mechanisms to manage the interaction and work among the participating organizations taking into account the dependencies and the shared resources among the processes.

Talking about B-ITa commonly leads one to think in aspects related to technology (IS architecture) and processes (process architecture). However, this is a misunderstanding when talking about B-ITa in CNOs. In collaborative B-ITa projects, beside those 'easily perceived' aspects, coordination and partnering structure are vital for assuring the success of such projects [19]. These two B-ITa domains are general domains in the sense that they are not directly related to B-ITa but they help to create an environment where B-ITa improvements can be easily achieved. If coordination and partnering structure are not considered in collaborative B-ITa projects, B-ITa efforts could turn more complex than they already are. In our case studies, we have found that these two domains affect B-ITa in CNOs. The case of IBM and its joint development alliance consortium (which includes Siemens, Samsung, Infineon, and STMicroelectronics), to develop semiconductor technologies (http://www.ibm.com), also supports our findings. As consortia often do it, this consortium conceded to one participating organization (IBM) the right to organize the consortium and to determine the behaviors of the other participants within a given context to coordinate their actions, to speak on everybody's behalf and to exert leadership, i.e., to use authority.

\section{The ICoNOs MM}

Table 2 presents the ICoNOs MM. The first column in the table represents the B-ITa levels. The cells of the ICoNOs MM contain B-ITa process areas grouped in domains. A process area is a group of practices in a domain which, when implemented collectively, satisfy goals considered important for making an improvement in that domain (e.g., a process area in the IS architecture domain is 'IS portfolio management'). We make the explicit note that, when finalizing the development of the ICoNOs MM, we have changed the position of some of the process areas presented in the previous version of the MM [18]. We found that some of the process areas are strongly related to each other. We then decided to re-arrange de position of them, so that some of the original process areas are now goals of, or practices in, another process area.

Similarly to CMMI, these process areas have specific and generic goals, which the activities in each process area are supposed to achieve. Specific goals describe characteristics that must be present to satisfy a particular process area. That is, they are specific for this area. In contrast, generic goals apply to all process areas, although their instantiation for each process area can differ. For example, a CMMI generic goal is 
Table 2. The ICoNOs MM

\begin{tabular}{|l|l|l|l|l|}
\cline { 2 - 5 } \multicolumn{2}{|c|}{ Partnering structure } & \multicolumn{1}{c|}{ IS architecture } \\
\hline $\mathbf{5}$ & \multicolumn{2}{|l|}{} & $\begin{array}{l}\text { Inter-organizational IS arch. optimization } \\
\text { Risk analysis and mitigation }\end{array}$ & $\begin{array}{l}\text { IoAO } \\
\text { RAM }\end{array}$ \\
\hline $\mathbf{4}$ & Metric-based roles exploration & MRE & Quantitative IS portfolio management & QPM \\
\hline $\mathbf{3}$ & $\begin{array}{l}\text { Governance structure and compliance } \\
\text { Service level agreements definition }\end{array}$ & GSC & $\begin{array}{l}\text { IS requirements management } \\
\text { IS capabilities definition } \\
\text { IS portfolio management }\end{array}$ & $\begin{array}{l}\text { IsRM } \\
\text { IsCD } \\
\text { IsPM }\end{array}$ \\
\hline $\mathbf{2}$ & Business model definition & BMD & Current IS architecture description & CSA \\
\hline $\mathbf{1}$ & \multicolumn{2}{|l}{} & & \\
\hline
\end{tabular}

\begin{tabular}{|c|c|c|c|c|}
\hline & \multicolumn{2}{|l|}{ Process architecture } & \multicolumn{2}{|l|}{ Coordination } \\
\hline 5 & Inter-organizational process optimization & $\mathrm{IoPO}$ & & \\
\hline 4 & $\begin{array}{l}\text { Organizational process performance } \\
\text { Event logs formal consistency }\end{array}$ & $\begin{array}{l}\mathrm{OPP} \\
\mathrm{EFC}\end{array}$ & Quantitative coordination relation analysis & QRA \\
\hline 3 & $\begin{array}{l}\text { Organizational process focus planning } \\
\text { Target process architecture formulation }\end{array}$ & $\begin{array}{l}\text { PFP } \\
\text { TPA }\end{array}$ & $\begin{array}{l}\text { Standardization } \\
\text { Communication-oriented coordination }\end{array}$ & $\begin{array}{l}\text { STD } \\
\text { COC }\end{array}$ \\
\hline 2 & Current process architecture description & $\overline{\mathrm{CPD}}$ & $\begin{array}{l}\text { Informal communication adjustment } \\
\text { Direct supervision }\end{array}$ & $\begin{array}{l}\text { InCA } \\
\text { DTS }\end{array}$ \\
\hline
\end{tabular}

'the process is institutionalized as a defined process'. This goal can be applied to all processes. Our ICoNOs MM incorporates the generic goals of CMMI and, therefore, we do not elaborate on them. The specific and generic goals are respectively decomposed in specific and generic practices. Practices describe what a CNO may implement to achieve the goals. These practices are expected and not mandatory. This means that one can implement alternative practices in substitution for the specific and generic practices that the MM includes. The only condition is that the goals must be satisfied, to perform a process, to reach a specific maturity level.

We conducted an exhaustive literature review in order to define the specific goals and the specific practices for each of the B-ITa process areas. However, due to space constraints, in this section, we only list the specific goals and the specific practices of the B-ITa process areas included in the domains of IS architecture and process architecture. The list of specific goals and specific practices of the other two domains can be found in [19]. We only introduce the specific goals and specific practices included in levels two and three because in these levels is where CNOs can make the most significant improvements. In the following, the acronyms SG and SP stand for specific goal and specific practice, respectively.

- Current IS architecture description (CSA). The main purpose of the CSA is to create a snapshot of the existing ISs and data, assessing what the current status of the $\mathrm{CNO}$ is concerning ISs.

SG1 Characterize the existing software applications.

SP1.1 Make an inventory of the current ISs of the entire CNO.

SP1.2 Describe what the ISs do (not how they do it) and their status. 
SG2 Build the software application model.

SP2.1 Define the stakeholders (owners/users) of the ISs.

SP2.2 Identify the organizational units supported.

SP2.3 Identify the processes supported.

SP2.4 Illustrate how data flows throughout the ISs.

SP2.5 Describe the precedent and successor ISs.

SG3 Define the IS standards and principles.

- IS capabilities definition (IsCD). The main purpose of the IsCD is to define the ability of the CNO to operate efficiently by using ISs to achieve congruence with the business environment where it works and to improve its performance.

SG1 Analyze the IT and business skills of the participants in the CNO.

SG2 Identify the motivating values of the participants.

SG3 Define the IS capabilities of the CNO considering the previous two SGs.

- IS portfolio management (IsPM). The main purpose of the IsPM is to create the right mix of ISs investments to properly use limited resources while providing the maximum business benefit.

SG1 Define the target IS architecture.

SP1.1 Identify improvements and adjustments based on the requirements.

SP1.2 Build the target IS model.

SP1.3 Verify and validate the target IS architecture (gap analysis).

SP1.4 Define the new investments on ISs.

SG2 Define insights and facts for decision making.

SP2.1 Define key application metrics.

SP2.2 Conduct risk analysis.

SG3 Develop a plan to obtain all participants' buy-in.

- IS requirements management (IsRM). The main purpose of the IsRM is to manage the changing IS requirements during their engineering process and the development of the required ISs.

SG1 Obtain an understanding of IS requirements.

SP1.1 Identify and record requirements.

SP1.2 Assess the impact of requirements.

SP1.3 Determine requirements priorities.

SG2 Obtain commitment to requirements of all participants.

SG3 Define policies for requirements management.

SG4 Use a database to manage requirements.

- Current process architecture description (CPD). The main purpose of the CPD is to create a snapshot of the existing processes, and maintaining a repository of measures and assets.

SG1 Characterize the existing processes.

SP1.1 Make an inventory of the current relevant processes.

SP1.2 Build a process model. 
SG2 Establish the CNO's measurement repository.

SP2.1 Determine the needs for storing, retrieving and analyzing measurements.

SP2.2 Define and agree upon a common set of process measures.

SP2.3 Design and implement the measurement repository.

SP2.4 Make the contents of the repository available for use by the CNO.

SG3 Establish the CNO's process asset library.

SP3.1 Design and implement the organization's process asset library.

SP3.2 Specify the criteria for including items in the library.

SP3.3 Specify the procedures for storing and retrieving items.

SP3.4 Have the selected items entered into the library.

SP3.5 Make the items available for use by the CNO.

- Organizational process focus planning (PFP). The main purpose of the PFP is to plan, implement, and deploy process improvements based on the strengths and weaknesses of the CNO's processes and assets.

SG1 Determine process improvement opportunities.

SP1.1 Establish the CNO process needs.

SP1.2 Appraise the CNO's processes.

SP1.3 Identify the CNO's process improvements.

SG2 Plan and implement process improvements.

SP2.1 Establish process action plans.

SP2.2 Implement process action plans.

SG3 Deploy CNO process assets and incorporate lessons learnt.

SP3.1 Deploy CNO process assets.

SP3.2 Monitor implementation.

SP3.3 Incorporate lessons learnt into the $\mathrm{CNO}$ process assets.

- Target process architecture formulation (TPA). The main purpose of the TPA is to evaluate, select and design processes needed to support the desired to-be state of the process architecture.

SG1 Establish general tailoring guidelines.

SG2 Identify external interfaces.

SG3 Gather information about services/products offerings.

SG4 Define the mayor activities to support the services/products offerings.

SG5 Build a target process model.

\section{Conclusion}

In this paper, we have presented a MM for assessing the maturity of B-ITa in CNOs: the ICoNOs MM. This model is a promising attempt to properly understand the B-ITa domains involved in collaborative B-ITa in terms of process maturity.

Although the ICoNOs MM is almost finalized, we stress that the model needs to be further revised, and eventually modified. At this moment, we have finalized a 
pilot assessment in a CNO composed by three Dutch universities, where we used the ICoNOs MM to appraise the maturity of its B-ITa. After the assessment, we asked to the participants in the CNO how they perceived the assessment process, the model, and the results. This is helping us to prove the usability of the ICoNOs MM in a reallife context, and to eventually produce an improved version of the model.

\section{References}

1. Chan, Y., Reich, B.: IT alignment: What have we learned? Journal of Inf. Tech. 22(4), 297-315 (2007)

2. Kearns, G.S., Lederer, A.L.: The effect of strategic alignment on the use of IS-based resources for competitive advantage. Journal of Strategic Inf. Systems 9(4), 265-293 (2000)

3. Powell, T.: Organizational alignment as competitive advantage. Strategic Management Journal 13(2), 119-134 (1992)

4. Floyd, S.W., Wooldridge, B.: Path analysis of the relationship between competitive strategy, information technology, and financial performance. Journal of Mngmt. Inf. Systs. 7(1), 47-64 (1990)

5. de Koning, D., van der Marck, P.: IT Zonder Hoofdpijn: Een Leidraad voor het Verbeteren van de Bedrijfsprestaties. Prentice Hall, Englewood Cliffs (2002) (in Dutch)

6. Duffy, J.: Maturity models: Blueprints for e-volution. Strategy \& Leadership 29(6), 19-26 (2001)

7. Luftman, J.N.: Assessing IT-business alignment. Inf. Systems. Mngmt. 20(4), 9-15 (2003)

8. Sanchez Ortiz, A.: Testing a model of the relationships among organizational performance, IT-business alignment and IT governance. PhD thesis, University of North Texas, Denton, Texas, USA (2003)

9. Camarinha-Matos, L.M., Afsarmanesh, H.: The emerging discipline of collaborative networks. In: Virtual Enterprises and Collaborative Networks. Kluwer Academic Pub., Dordrecht (2004)

10. Santana Tapia, R.: What is a networked business? Technical Report TR-CTIT-06-23a, University of Twente, Enschede, The Netherlands (2006)

11. Santana Tapia, R., van Eck, P., Daneva, M.: Validating the domains of an interorganizational business-IT alignment assessment instrument. Technical Report TR-CTIT-08-53 University of Twente, Enschede, The Netherlands (2008)

12. Santana Tapia, R., Daneva, M., van Eck, P., Castro Cárdenas, N., van Oene, L.: BusinessIT alignment domains and principles for networked organizations: A qualitative multiple case study. In: OTM 2008, Part II, pp. 241-252. Springer, Heidelberg (2008)

13. Santana Tapia, R., van Oene, L.: Some empirical evidence on business-IT alignment processes in the public sector: A case study report. Technical Report TR-CTIT-08-46 University of Twente, Enschede, The Netherlands (2008)

14. Santana Tapia, R.: Converging on business-IT alignment best practices: Lessons learned from a Dutch cross-governmental partnership. To appear in the 15th International Conference on Concurrent Enterprising (2009)

15. Santana Tapia, R.: A value-based maturity model for IT alignment in networked businesses. In: Workshops and Doctoral Consortium of the 18th Int. Conf. on Advanced Information Systems Engineering (CAISE 2006), pp. 1201-1208. Presses Universitaires de Namur (2006) 
16. Santana Tapia, R., Daneva, M., van Eck, P.: Validating adequacy and suitability of business-IT alignment criteria in an inter-enterprise maturity model. In: The 11th IEEE Int. EDOC Enterprise Computing Conference, pp. 202-213. IEEE Computer Society Press, Los Alamitos (2007)

17. Santana Tapia, R., Daneva, M., van Eck, P.: Developing an inter-enterprise alignment maturity model: Research challenges and solutions. In: The 1st International Conference on Research Challenges on Information Science (RCIS 2007), pp. 51-59 (2007)

18. Santana Tapia, R., Daneva, M., van Eck, P., Wieringa, R.: Towards a business-IT alignment maturity model for collaborative networked organizations. In: The International Workshop on Enterprise Interoperability (IWEI 2008), CTIT, pp. 70-81 (2008)

19. Santana Tapia, R.: Coordination and partnering structure are vital domains in collaborative business-IT alignment: Elaborating on the ICoNOs MM. To appear in the 2009 IFIP/IEEE International Symposium on Integrated Network Mngmt. - Workshops Proceedings (2009)

20. Maier, M.W., Emery, D., Hilliard, R.: Software architecture: Introducing IEEE standard 1471. Computer 34(4), 107-109 (2001) 